Article

\title{
An Approximation Theorem for Vector Equilibrium Problems under Bounded Rationality
}

\author{
Wensheng Jia, Xiaoling Qiu * and Dingtao Peng \\ School of Mathematics and Statistics, Guizhou University, Huaxidadao, Guiyang 550025, China; \\ wsjia@gzu.edu.cn (W.J.); dtpeng@gzu.edu.cn (D.P.) \\ * Correspondence: xlqiu@gzu.edu.cn
}

Received: 1 December 2019; Accepted: 23 December 2019; Published: 1 January 2020

\begin{abstract}
In this paper, our purpose is to investigate the vector equilibrium problem of whether the approximate solution representing bounded rationality can converge to the exact solution representing complete rationality. An approximation theorem is proved for vector equilibrium problems under some general assumptions. It is also shown that the bounded rationality is an approximate way to achieve the full rationality. As a special case, we obtain some corollaries for scalar equilibrium problems. Moreover, we obtain a generic convergence theorem of the solutions of strictly-quasi-monotone vector equilibrium problems according to Baire's theorems. As applications, we investigate vector variational inequality problems, vector optimization problems and Nash equilibrium problems of multi-objective games as special cases.
\end{abstract}

Keywords: vector equilibrium problems; approximation theorem; bounded rationality; generic convergence

\section{Introduction}

In the last two decades, the vector equilibrium problem (VEP) has received much attention because it provides a unified framework for many important particular problems such as vector variational inequality, vector optimization, vector saddle points, multiobjective games, multiobjective transportation equilibrium problems, and so forth. A large amount of research on VEP mainly focuses on the following aspects-the existence of solution (see References [1-6]), property of solution (including connection and continuity, see References [7-12]), stability of solution (including well-posedness and sensitivity analysis, see References [13-17]) and various algorithms for solving VEP (see References [6,18-22] and the references therein). However, the approximation theorem of VEP has hardly been seen from the perspective of algorithms.

As we know, the approximation theorems are central to many computing problems and the related theory provides insight as well as a foundation for algorithms. Various iterative methods for solving equilibrium problems have been developed, which can be divided into several classes-fixed point methods, extragradient methods, descent methods, proximal point and Tykhonov-Browder regularization methods (see the recent survey in Reference [6]). On the one hand, we know that an exact solution of a VEP may not exist in practice if the data of the VEP model are not "regular" and so the VEP model is generally solved by numerical methods (iterative procedures or heuristic algorithms), producing approximations to the exact solutions. Thus, in general, obtaining exact solutions to many practical problems is not possible. Naturally, it is interesting and important to investigate some characterizations for approximate solutions of VEP. On the other hand, from the view of Simon's bounded rationality theory [23], the decision is to seek the satisfactory solution rather than the exact optimal solution. Indeed, the satisfactory solution corresponds to the approximate solution, which reflects the bounded rationality and the exact optimal solution corresponds to the full rationality. However, the exact optimal solution is hardly obtained in the specific calculation process. We always 
obtain the approximate solution by means of iterative algorithms. Therefore, it is very important that the approximate solution approaches the exact optimal solution which implies that the full rationality can be approached by the bounded rationality.

In this paper, we aim to give an approximation theorem for VEP under some general assumptions and frames. Some corollaries and remarks will be obtained under some conditions. This paper is organized as follows-in Section 2, some definitions and two necessary lemmas are introduced. In Section 3, an approximation theorem and some corollaries are proved, and we give the details proofs and notations. In Section 4, the generic convergence theorem on monotone VEP is obtained. Some applications of the approximation theorem for vector equilibrium problems are given in Section 5 . Section 6 provides a conclusion.

\section{Preliminaries}

Definition 1 ([24]). Let $X$ be a Hausdorff topological space and $\phi: X \rightarrow R$ be a function.

(i) $\phi$ is called upper pseudocontinuous (lower pseudocontinuous) at $x_{0} \in X$ iff for all $x \in X$ such that $\phi\left(x_{0}\right)<\phi(x)\left(\phi(x)<\phi\left(x_{0}\right)\right)$, we have

$$
\limsup _{y \rightarrow x_{0}} \phi(y)<\phi(x) \quad\left(\phi(x)<\liminf _{y \rightarrow x_{0}} \phi(y)\right) .
$$

(ii) $\phi$ is called pseudocontinuous at $x \in X$ iff $\phi$ is both upper pseudocontinuous and lower pseudocontinuous at $x$.

(iii) Let $\Phi: X \rightarrow R^{k}$ be a vector-valued mapping, where $\Phi=\left\{\phi_{1}, \ldots, \phi_{k}\right\}$. Then $\Phi$ is $R_{+}^{k}-$ pseudocontinuous (resp.upper pseudocontinuous, lower pseudocontinuous) iff $\phi_{i}$ is pseudocontinuous (resp.upper pseudocontinuous, lower pseudocontinuous) for every $i=1, \ldots, k$.

Remark 1. Each upper(resp.lower) semicontinuous function is also upper(resp.lower) pseudocontinuous. But the converse is not true.

$$
\text { Let } X=[0,2], \phi_{i}: X \rightarrow R, i=1,2 \text {, }
$$

$$
\phi_{1}(x)=\left\{\begin{array}{ll}
1-x, & 0 \leq x<1, \\
-1, & 1 \leq x \leq 2 .
\end{array} ; \phi_{2}(x)= \begin{cases}x-1, & 0 \leq x<1 \\
2, & 1 \leq x \leq 2 .\end{cases}\right.
$$

It is obvious that $\phi_{1}$ is not upper semicontinuous but upper pseudocontinuous at $x=1$ and that $\phi_{2}$ is not lower semicontinuous but lower pseudocontinuous at $x=1$.

Definition 2 ([14]). A vector-valued function $\phi: X \times X \rightarrow R^{k}$ is called $R^{k}$-strictly-quasi-monotone on $X \times X$ iff for any $x, y \in X$ with $x \neq y, \phi(x, y) \in-i n t R^{k}$ implies $\phi(y, x) \in-R^{k}$.

Let $(X, d)$ be a metric space, the following two lemmas are important to prove the approximation theorem for VEP in Section 3.

Lemma 1 ([25]). Assume that there is a sequence $\left\{A_{n}\right\}$ of a nonempty bounded subset of $X$ and a nonempty compact subset $A$ of $X$. If $x_{n} \in A_{n}$ for any $n=1,2, \cdots$ and $h\left(A_{n}, A\right) \rightarrow 0$, where $h\left(A_{n}, A\right)$ means the Hausdorff metric on $X$, then there exists a subsequence $\left\{x_{n_{k}}\right\}$ of $\left\{x_{n}\right\}$ such that $x_{n_{k}} \rightarrow x^{*} \in A$.

Lemma 2 ([25]). Assume that there is a sequence $\left\{A_{n}\right\}$ of a nonempty bounded subset of $X$ and a nonempty subset $A$ of $X$. Let $G$ be an open set of $X$, if $A \cap G \neq \varnothing$ and $h\left(A_{n}, A\right) \rightarrow 0$, then there exists a positive integer $N$ such that for any $n \geq N, A_{n} \cap G \neq \varnothing$. 


\section{Approximation Theorem}

In this section, we are interested in the approximation of both the constraint set and the bifunction of (VEP). To this purpose, we introduce a definition of an approximate equilibrium point. We start our analysis with (VEP).

Let $X$ be a nonempty subset of $R^{n}$, let $A$ be a nonempty compact subset of $X$ and let $\phi: X \times X \rightarrow$ $R^{k}$ be defined by $\phi(x, y)=\left(\phi_{1}(x, y), \cdots, \phi_{k}(x, y)\right), \phi_{i}: X \times X \rightarrow R$ for all $x, y \in X$ where each function $\phi_{i}$ is pseudocontinuous and satisfies the condition $\phi_{i}(x, x)=0$ for all $x \in X$.

Problem (VEP) consists in finding $x^{*} \in X$ such that

$$
\phi\left(x^{*}, y\right) \notin-i n t R_{+}^{k}, \forall y \in X .
$$

Definition 3. Given $\phi: X \times X \rightarrow R^{k}$ and $\epsilon=\left(\epsilon_{1}, \epsilon_{2}, \cdots, \epsilon_{k}\right), \epsilon_{i}>0, i=1,2, \cdots, k, x^{*}$ is said to be an $\epsilon-$ equilibrium point of $\phi$ if

$$
\phi\left(x^{*}, y\right)+\epsilon b \notin-i n t R_{+}^{k}, \forall y \in X
$$

where $b=(1,1, \cdots, 1)^{T}$.

For solving this problem, we propose approximating the function $\phi$ by a sequence of vector-valued functions $\left\{\phi^{n}\right\}$ such that for all $n$,

$$
\phi^{n}: X \times X \rightarrow R^{k}
$$

and

$$
\sup _{(x, y) \in X \times X}\left\|\phi^{n}(x, y)-\phi(x, y)\right\| \rightarrow 0 \quad(n \rightarrow \infty)
$$

Furthermore, the compact subset $A$ is approximated by means of a sequence $\left\{A_{n}\right\}$ of subsets of $X$ with

$$
h\left(A_{n}, A\right) \rightarrow 0 \quad(n \rightarrow \infty)
$$

where $h\left(A_{n}, A\right)$ denotes the Hausdorff metric on $X$ between the sets $A_{n}$ and $A$.

Theorem 1. Let $\left\{x_{n}\right\}$ be a sequence in $X$ and let $\left\{\epsilon_{n}\right\}$ be a sequence of positive numbers such that for all $n$ and $y \in A_{n}$,

$$
\phi^{n}\left(x_{n}, y\right)+\epsilon_{n} b \notin-i n t R_{+}^{k}
$$

where $b=(1,1, \cdots, 1) \in R^{k}$. If $d\left(x_{n}, A_{n}\right) \rightarrow 0$ and $\left\{\epsilon_{n}\right\} \rightarrow 0$ as $n \rightarrow \infty$, then

(i) There exists a subsequence $\left\{x_{n_{j}}\right\}$ of $\left\{x_{n}\right\}$ which converges to some $x^{*} \in A$;

(ii) For any $y \in A, \phi\left(x^{*}, y\right) \notin-i n t R_{+}^{k}$.

Proof. (i) Since $d\left(x_{n}, A_{n}\right) \rightarrow 0(n \rightarrow \infty)$, there exists a sequence $x_{n}^{\prime} \in A_{n}$ such that $d\left(x_{n}, x_{n}^{\prime}\right) \rightarrow 0$. Since the compactness of $A$ and (6), by Lemma 1, there exists a subsequence $x_{n_{k}}^{\prime}$ of $x_{n}^{\prime}$ such that $x_{n_{k}}^{\prime} \rightarrow x^{*} \in A$. Hence there exists a subsequence $\left\{x_{n_{j}}\right\}$ of $\left\{x_{n}\right\}$ which converges to some $x^{*} \in A$.

(ii) Without loss of generality, it follows from (i) that we may assume that $x_{n} \rightarrow x^{*}$. If the statement of (ii) was false, then there exists some point $y_{0} \in A$ such that $\phi\left(x^{*}, y_{0}\right) \in-i n t R_{+}^{k}$. Since $\phi\left(x^{*}, x^{*}\right)=0$, then $\forall i=1,2, \cdots, k, \phi_{i}\left(x^{*}, y_{0}\right)<\phi_{i}\left(x^{*}, x^{*}\right)=0$. By the definition of pseudocontinuity of $\phi_{i}$ at $\left(x^{*}, y_{0}\right)$, for each $i=1,2, \cdots, k$, we have

$$
\limsup _{x^{\prime} \rightarrow x^{*}, y^{\prime} \rightarrow y_{0}} \phi_{i}\left(x^{\prime}, y^{\prime}\right)<\phi_{i}\left(x^{*}, x^{*}\right)=0 \text {. }
$$


Hence there exists $\sigma_{0}>0$ such that

$$
\limsup _{x^{\prime} \rightarrow x^{*}, y^{\prime} \rightarrow y_{0}} \phi_{i}\left(x^{\prime}, y^{\prime}\right)<-\sigma_{0} .
$$

By Proposition 2.1 of Reference [24], there exists a neighborhood $O\left(x^{*}\right)$ of $x^{*}$ and a neighborhood $O\left(y_{0}\right)$ of $y_{0}$ such that $\phi_{i}\left(x^{\prime}, y^{\prime}\right)<-\sigma_{0}$ for any $x^{\prime} \in O\left(x^{*}\right)$ and any $y^{\prime} \in O\left(y_{0}\right)$.

Notice that the condition (5) and $\epsilon_{n} \rightarrow 0(n \rightarrow \infty)$, there exists a positive integer $N_{1}$ such that for any $n \geq N_{1}$,

$$
\sup _{(x, y) \in X \times X}\left\|\phi^{n}(x, y)-\phi(x, y)\right\|<\frac{\sigma_{0}}{2}, \quad \epsilon_{n}<\frac{\sigma_{0}}{2} .
$$

Since $n \rightarrow \infty, x_{n} \rightarrow x^{*}, h\left(A_{n}, A\right) \rightarrow 0$ and $y_{0} \in A$, by Lemma 2 , there exists a positive integer $N_{2} \geq N_{1}$ such that for any $n \geq N_{2}, x_{n} \in O\left(x^{*}\right)$ and $O\left(y_{0}\right) \cap A_{n} \neq \varnothing$. We take $y_{n} \in O\left(y_{0}\right) \cap A_{n}$. Hence we have

$$
\phi_{i}\left(x_{n}, y_{n}\right)<-\sigma_{0}
$$

and

$$
\phi_{i}^{n}\left(x_{n}, y_{n}\right)<\phi_{i}\left(x_{n}, y_{n}\right)+\frac{\sigma_{0}}{2}<-\frac{\sigma_{0}}{2}<-\epsilon_{n} .
$$

Consequently $\phi^{n}\left(x_{n}, y_{n}\right)+\epsilon_{n} b \in-i n t R_{+}^{k}$. This leads to a contradiction that $x_{n}$ is an $\epsilon_{n}$-equilibrium point of $\phi^{n}$. Thus the proof is finished.

Remark 2. In Theorem 1 , if $\phi_{i}$ is continuous for each $n=1,2,3, \cdots$, then Theorem 1 holds; if $x_{n} \in A_{n}$ for each $n=1,2,3, \cdots$, then Theorem 1 also holds.

Remark 3. It is obvious that Theorem 1 reflects the theoretical significance-the bifunctions are approximate, the constraint sets are approximate, and the solutions of VEP are also approximate. In that case, there exists $\left\{x_{n_{k}}\right\} \subset\left\{x_{n}\right\}$ such that $x_{n_{k}} \rightarrow x^{*} \in A$. If we see the solutions of $\phi$ as the optimal solutions under full rationality and $\epsilon_{n}$-equilibrium points of $\phi^{n}$ as the approximate solutions under bounded rationality, Theorem 1 reflects that the full rationality can be approached by the bounded rationality.

Notice that the above approximation theorem for vector equilibrium problems can be weakened by the following Corollary 1.

Corollary 1. Let $X$ be a nonempty subset of $R^{n}$ and all the following assumptions are satisfied:

(i) For any $n=1,2, \cdots$, the vector-valued function sequence $\phi^{n}: X \times X \rightarrow R^{k}$ is satisfied with sup $\left\|\phi^{n}(x, y)-\phi(x, y)\right\| \rightarrow 0$, where the function $\phi: X \times X \rightarrow R^{k}$ is upper pseudocontinuous $(x, y) \in X \times X$ at the first variable on $X$;

(ii) $A$ is a nonempty compact subset of $X$ and $\phi(x, x)=0$ for any $x \in A$;

(iii) For any $n=1,2, \cdots, x_{n} \in A$ is $\epsilon_{n}$-equilibrium point of $\phi^{n}$, that is, $\phi^{n}\left(x_{n}, y\right)+\epsilon_{n} b \notin-i n t R_{+}^{k}$ for any $y \in A$, where $\epsilon_{n}>0$ and $\epsilon_{n} \rightarrow 0(n \rightarrow \infty)$.

Then

(i) there exists a subsequence $\left\{x_{n_{j}}\right\}$ of $\left\{x_{n}\right\}$ which converges to some $x^{*} \in A$;

(ii) for any $y \in A, \phi\left(x^{*}, y\right) \notin-i n t R_{+}^{k}$.

Proof. In Theorem 1 , let $A_{n}=A, n=1,2, \cdots$, then Corollary 1 can be completed in the similar method as shown in Theorem 1. 
For scalar equilibrium problems, we can obtain the following Corollary 2, which can be seen Theroem A in Reference [26].

Corollary 2. Let X be a metric space and all the following assumptions are satisfied:

(i) For any $n=1,2, \cdots$, the function sequence $f^{n}: X \times X \rightarrow R$ is satisfied with $\sup _{(x, y) \in X \times X} \mid f^{n}(x, y)-$ $f(x, y) \mid \rightarrow 0$, where the function $f: X \times X \rightarrow R$ is upper pseudocontinuous;

(ii) For any $n=1,2, \cdots, A_{n}$ is a subset sequence of $X$ with $h\left(A_{n}, A\right) \rightarrow 0(n \rightarrow \infty)$, where $A$ is a nonempty compact subset of $X$ and $f(x, x)=0$ for any $x \in A$;

(iii) For any $n=1,2, \cdots, x_{n} \in A_{n}$ is $\epsilon_{n}$-equilibrium point of $f^{n}$, that is, $f^{n}\left(x_{n}, y\right) \geq-\epsilon_{n}$ for any $y \in A_{n}$, where $\epsilon_{n}>0$ and $\epsilon_{n} \rightarrow 0(n \rightarrow \infty)$.

Then

(i) there exists a subsequence $\left\{x_{n_{j}}\right\}$ of $\left\{x_{n}\right\}$ which converges to some $x^{*} \in A$;

(ii) for any $y \in A, f\left(x^{*}, y\right) \geq 0$.

\section{Generic Convergence}

In this section, we will study the generic convergence of VEP. Firstly we construct a space of VEP under some assumptions and study the property of the set of solutions.

Let a space $\mathrm{M}$ of VEP be defined by

$$
M=\left\{u=(\phi, A): \quad \begin{array}{l}
\phi: X \times X \rightarrow R^{k} \text { is } R^{k}-\text { upper-semicontinuous on } X \times X ; \\
\phi \text { is } R^{k}-\text { strictly }- \text { quasi-monotone on } X \times X ; \\
\text { A is a monempty compact subset of } X ; \\
\text { and } \exists x^{*} \in A \text { such that } \phi(x, y) \notin-i n t R_{+}^{k}, \forall y \in A .
\end{array}\right\}
$$

For any $U_{1}=\left(\phi_{1}, A_{1}\right), U_{2}=\left(\phi_{2}, A_{2}\right)$, denote

$$
\rho\left(U_{1}, U_{2}\right)=\sup _{(x, y) \in X \times X}\left\|\phi_{1}(x, y)-\phi_{2}(x, y)\right\|+h\left(A_{1}, A_{2}\right),
$$

where $h$ is the Hausdorff distance on $X$.

Denote by $S(u)$ the set of all solutions to VEP in A. Then it yields a set-valued mappings $S: M \rightarrow 2^{X}$, that is, $S(u)=\left\{x \in A: \phi(x, y) \notin-i n t R_{+}^{k}, \forall y \in A\right\}$ for all $u \in M$.

By Reference [14], the authors obtain an important result using the way of set-valued analysis.

Lemma 3. There exists a dense everywhere residual subset $Q$ of $M$ such that $S(u)=\{x \in A: \phi(x, y) \notin$ $\left.-i n t R_{+}^{k}, \forall y \in A\right\}$ is a singleton for each $u=(\phi, A) \in Q$, that is, VEP has a unique solution in $A$.

Lemma 4. If the solution of VEP is a singleton set, then $\left\{x_{n}\right\} \rightarrow x^{*}$.

Proof. By contradiction, assume that $\left\{x_{n}\right\}$ does not converge to $x^{*}$, then there exists $\delta>0$ and a subsequence $\left\{x_{n_{k}}\right\}$ of $\left\{x_{n}\right\}$ such that $d\left(x^{*}, x_{n_{k}}\right) \geq \delta, \forall k$. By Theorem 1 , there exists a subsequence $\left\{x_{n_{k_{t}}}\right\}$ of $\left\{x_{n_{k}}\right\}, x_{n_{k_{t}}} \rightarrow \bar{x} \in A$, we may suppose that $x_{n_{k}} \rightarrow \bar{x}$, that is $d\left(x_{n_{k}}, \bar{x}\right) \rightarrow 0$. $\bar{x}$ satisfies $\phi(\bar{x}, y) \notin-i n t R_{+}^{k}$ for each $y \in A$. Since the solution is singleton set, then $\bar{x}=x^{*}, d\left(\bar{x}, x_{n_{k}}\right) \geq \delta$ which is a contradiction. Thus, the result holds.

Remark 4. In fact, the convergence of Lemma 4 is better than Theorem 1, Corollary 1 and Corollary 2. In Lemma 4, there exists the convergence sequence $x_{n} \rightarrow x^{*} \in A$ but not the convergence subsequence $x_{n_{j}} \rightarrow x^{*} \in A$ if the solution of VEP is a singleton set.

Next, we can obtain the generic convergence theorem of solutions of VEP. 
Theorem 2. Let $\left\{x_{n}\right\}$ be $\epsilon_{n}$ - solution of $\phi^{n}$ with the convergence function $\phi$ under the uniform metric. There exists a dense residual subset $Q$ of $M$ such that for any $u=(\phi, A) \in Q$, then $\phi$ has a unique solution $x^{*}$ with $\left\{x_{n}\right\} \rightarrow x^{*}$ and $\phi\left(x^{*}, y\right) \notin-i n t R_{+}^{k}, \forall y \in A$.

Proof. It is obvious that Theorem 2 can be deduced by combining Lemma 4 with Lemma 3.

Remark 5. Note that $\phi: X \times X \rightarrow R^{k}$ is $R^{k}$-upper-continuouson $X \times X$. If $\phi: X \times X \rightarrow R^{k}$ is $R^{k}$-pseudocontinuous on $X \times X$, then Theorem 2 also holds. All the proofs are similar and so we omit it.

\section{Applications}

In this section, we obtain some applications of the approximation theorem for vector equilibrium problems.

\subsection{Vector Variational Inequality Problems}

Let $X$ and $Y$ be topological vector spaces and $K$ be a nonempty subset of $X$. Let $T: K \rightarrow L(X, Y)$ be an operator, for each $l \in L(X, Y)$, the value of $l$ at $x$ is denoted by $\langle l, x\rangle$. The vector variational inequality problems (VVIPs) are defined as follows:

Find $x^{*} \in K$ such that $<T\left(x^{*}\right), y-x^{*}>\notin-i n t R_{+}^{k}$, for all $y \in K$.

Set

$$
\phi(x, y)=<T(x), y-x>, \forall x, y \in K
$$

Then VVIPs are equivalent to the corresponding VEPs. By Theorem 1, we can obtain the following Proposition 1.

Proposition 1. If the following conditions are satisfied:

(i) For any $n=1,2, \cdots$, the operator sequence $T^{n}: K \rightarrow L(X, Y)$ is satisfied with $\sup _{x \in K} \| T^{n}(x)-$ $T(x) \| \rightarrow 0$, where the operator $T: K \rightarrow L(X, Y)$ is pseudocontinuous;

(ii) For any $n=1,2, \cdots, A_{n}$ is a subset sequence of $K$ with $h\left(A_{n}, A\right) \rightarrow 0(n \rightarrow \infty)$, where $A$ is a nonempty compact subset of $K$;

(iii) For any $n=1,2, \cdots, x_{n} \in K$ and $d\left(x_{n}, A_{n}\right) \rightarrow 0(n \rightarrow \infty)$, there holds $\left\langle T^{n}\left(x^{n}\right), y-x^{n}\right\rangle+\epsilon_{n} b \notin$ - int $R_{+}^{k}, \forall y \in A_{n}$, where $b=(1,1, \cdots, 1) \in R_{+}^{k}, \epsilon_{n}>0$ and $\epsilon_{n} \rightarrow 0(n \rightarrow \infty)$.

Then

(i) there exists a subsequence $\left\{x_{n_{j}}\right\}$ of $\left\{x_{n}\right\}$ which converges to some $x^{*} \in A$;

(ii) for any $y \in K,<T\left(x^{*}\right), y-x^{*}>\notin-i n t R_{+}^{k}$.

\subsection{Vector Optimization Problems}

Let $X$ be a topological vector space and $K$ be a nonempty subset of $X$. Let $f: K \rightarrow R^{k}$ be a vector function. The vector optimization problems (VOPs) are defined as follows:

Find $x^{*} \in K$ such that $f(y)-f\left(x^{*}\right) \notin-i n t R_{+}^{k}$, for all $y \in K$.

Set

$$
\phi(x, y)=f(y)-f(x), \forall x, y \in K
$$

Then VOPs are equivalent to the corresponding VEPs. By Theorem 1, we can obtain the following Proposition 2.

Proposition 2. If the following conditions are satisfied: 
(i) For any $n=1,2, \cdots$, the function sequence $F^{n}: K \rightarrow R^{k}$ is satisfied with $\sup _{x \in K}\left\|F^{n}(x)-F(x)\right\| \rightarrow$ 0 , where the function $F: K \rightarrow R^{k}$ is pseudocontinuous;

(ii) For any $n=1,2, \cdots, A_{n}$ is a subset sequence of $K$ with $h\left(A_{n}, A\right) \rightarrow 0(n \rightarrow \infty)$, where $A$ is a nonempty compact subset of $K$;

(iii) For any $n=1,2, \cdots, x_{n} \in K$ and $d\left(x_{n}, A_{n}\right) \rightarrow 0(n \rightarrow \infty)$, there holds $F^{n}(y)-F^{n}\left(x_{n}\right)+\epsilon_{n} b \notin$ - int $R_{+}^{k}, \forall y \in A_{n}$, where $b=(1,1, \cdots, 1) \in R_{+}^{k}, \epsilon_{n}>0$ and $\epsilon_{n} \rightarrow 0(n \rightarrow \infty)$.

Then

(i) There exists a subsequence $\left\{x_{n_{j}}\right\}$ of $\left\{x_{n}\right\}$ which converges to some $x^{*} \in A$;

(ii) For any $y \in A, F(y)-F\left(x^{*}\right) \notin-i n t R_{+}^{k}$.

\subsection{Nash Equilibrium Problems of Multiobjective Games (In Short, NEPs)}

Let $N=\{1, \ldots, n\}$ denote the set of players. For each $i \in N$, assume that the strategy set of the $i$ th player is denoted by $K_{i}$. Now denote the players' strategy profile $x=\left(x_{i}, x_{-i}\right) \in K=\prod_{i \in N} K_{i}$, where $x_{i} \in K_{i}$ and $x_{-i} \in K_{-i}=\prod_{j \in N, j \neq i} K_{j}$.

For each $i \in N$, let $\Phi_{i}=\left\{\phi_{1}^{i}, \phi_{2}^{i}, \ldots, \phi_{l}^{i}\right\}: K_{i} \times K_{-i} \rightarrow R^{k}$ denote the objective function of the $i$ th player. A point $x^{*}=\left(x_{i}^{*}, x_{-i}^{*}\right) \in K$ is called to be a weakly Pareto-Nash equilibrium of multiobjective games, that is, for each $i \in N, \Phi_{i}\left(x_{i}^{*}, x_{-i}^{*}\right)-\Phi_{i}\left(x_{i}, x_{-i}^{*}\right) \notin-i n t R_{+}^{k}$, for all $x_{i} \in K_{i}$.

Set

$$
\phi(x, y)=\sum_{i=i}^{n}\left[\Phi_{i}\left(x_{i}, x_{-i}\right)-\Phi_{i}\left(y_{i}, x_{-i}\right)\right], \forall x, y \in K
$$

Then NEPs are equivalent to the corresponding VEPs. By Theorem 1, we can obtain the following Proposition 3.

Proposition 3. If the following conditions are satisfied:

(i) For any $i \in N$, the function sequence $\Phi_{i}^{m}: K \rightarrow R^{k}$ is satisfied with $\sup _{x \in K}\left\|\Phi_{i}^{m}(x)-\Phi_{i}(x)\right\| \rightarrow$ $0(m \rightarrow \infty)$, where the operator $\Phi_{i}: K \rightarrow R^{k}$ is pseudocontinuous for each $i=1,2, \ldots, n$;

(ii) For any $i \in N, A_{i}^{m}$ is a subset sequence of $K_{i}$ with $h\left(A_{i}^{m}, A_{i}\right) \rightarrow 0(m \rightarrow \infty)$, where $A_{i}$ is a nonempty compact subset of $K_{i}$;

(iii) For any $m=1,2, \cdots, x_{m} \in K$ and $d\left(x_{m}, \prod_{i=1}^{n} A_{i}^{m}\right) \rightarrow 0(n \rightarrow \infty)$, there holds $\Phi_{i}^{m}\left(y_{i}, x_{-i}^{m}\right)-$ $\Phi_{i}^{m}\left(x_{i}^{m}, x_{-i}^{m}\right)+\epsilon_{n} b \notin-i n t R_{+}^{k}$, for any $i \in N, \forall y_{i} \in A_{i}^{m}$, where $b=(1,1, \cdots, 1) \in R^{k}, \epsilon_{n}>0$ and $\epsilon_{n} \rightarrow 0(n \rightarrow \infty)$.

Then

(i) There exists a subsequence $\left\{x_{m_{j}}\right\}$ of $\left\{x_{m}\right\}$ which converges to some $x^{*} \in \prod_{i=1}^{n} A_{i}$;

(ii) For any $y_{i} \in A_{i}, \Phi_{i}\left(y_{i}, x_{-i}^{*}\right)-\Phi_{i}\left(x_{i}^{*}, x_{-i}^{*}\right) \notin-i n t R_{+}^{k}$ for each $i=1,2, \ldots, n$.

\section{Conclusions}

In this paper, we propose an approximate theorem (Theorem 1) for vector equilibrium problems under bounded rationality. According to the theorem, we illustrate the Simon's bounded rationality theory from the perspective of the vector equilibrium problems. That means the bounded rationality is an approximate way to full rationality. In Theorem 1, we can obtain the convergent subsequence of approximate solutions on VEPs and prove that the limitation of the subsequence belongs to the set of solutions of objective functions. This theory provides a basis for the algorithm of the vector equilibrium problem. Moreover, we also get the generic convergence of solutions of strictly-quasi-monotone vector equilibrium problems in the sense of the Baire category. Some examples, such as VVIPs, VOPs and NEPs, are given to investigate our results. 
Author Contributions: Writing-original manuscript and Project management, W.J.; Methodology and formal analysis, X.Q.; Supervision and writing-review, D.P. All authors have read and agreed to the published version of the manuscript.

Funding: This research was funded by NSFC (Grant numbers 11561013, 11861020), the Foundation of Overseas Students for Science and Technology Activities (Grant No. [2015]15), the Foundation for Selected Excellent Project of Guizhou Province for High-level Talents Back from Overseas (Grant No. [2018]03)), the Science and Technology Foundation of Guizhou Province (Grant numbers QKH[2014]7643, QKH[2019]1123), the Foundation of Guizhou University (Grant numbers 201405, 201811).

Acknowledgments: The authors gratefully acknowledge Yu Jian for many useful discussion.

Conflicts of Interest: The authors declare no conflict of interest.

\section{References}

1. Ansari, Q.H.; Oettli, W.; Schldoger, D. A generalization of vector equilibria. Math. Methods Oper. Res. 1997, 46, 147-152. [CrossRef]

2. Ansari, Q.H.; Yao, J.C. An existence result for the generalized vector equilibrium problem. Appl. Math. Lett. 1999, 12, 53-56. [CrossRef]

3. Bianchi, M.; Hadjisavvas, N.; Schaible, S. Vector equilibrium problems with generalized monotone bifunctions. J. Optim. Theory Appl. 1997, 92, 527-542. [CrossRef]

4. Kassay, G.; Miholca, M. Existence results for vector equilibrium problems given by a sum of two functions. J. Glob. Optim. 2015, 63, 195-211. [CrossRef]

5. CAPǍTĂ, A. Existence results for globally efficient solutions of vector equilibrium problems via a generalized KKM principle. Acta Math. Sci. 2017, 37, 463-476. [CrossRef]

6. Giancarlo, B.; Marco, C.; Massimo, P.; Mauro, P. Existence and solution methods for equilibria. Eur. J. Oper. Res. 2013, 227, 1-11.

7. Ansari, Q.H.; Konnov, I.V.; Yao, J.C. Characterizations of solutions for vector equilibrium problems. J. Optim. Theory Appl. 2002, 113, 435-447. [CrossRef]

8. Gong, X.H. Connectedness of the solution sets and scalarization for vector equilibrium problems. J. Optim. Theory Appl. 2007, 133, 151-161. [CrossRef]

9. Colao, V.; López, G.; Marino, G.; Martín-Márquez, V. Equilibrium problems in Hadamard manifolds. J. Math. Anal. Appl. 2012, 388, 61-77. [CrossRef]

10. Anh, L.Q.; Khanh, P.Q.; Tam, T.N. Continuity of approximate solution maps of primal and dual vector equilibrium problems. Optim. Lett. 2012, 13, 201-211. [CrossRef]

11. Han, Y.; Huang, N.J. Some characterizations of the approximate solutions to generalized vector equilibrium problems. J. Ind. Manag. Optim. 2016, 12, 1135-1151. [CrossRef]

12. Li, S.J.; Li, X.B.; Wang, L.N.; Teo, K.L. The Holder continuity of solutions to generalized vector equilibrium problems. Eur. J. Oper. Res. 2009, 119, 334-338. [CrossRef]

13. Fang, Y.P.; Hu, R.; Huang, N.J. Well-posedness for equilibrium problems and for optimization problems with equilibrium constraints. Comput. Math. Appl. 2008, 55, 89-100. [CrossRef]

14. Peng, D.T.; Yu, J.; Xiu, N.H. The uniqueness and well-posedness of vector equilibrium problems with a representation theorem for the solution set. Fixed Point Theory Appl. 2014, 115, 1-13. [CrossRef]

15. Peng, D.T.; Yu, J.; Xiu, N.H. Generic uniqueness of solutions of several types of nonlinear problems. Acta Math. Sin. Chin. Ser. 2014, 57, 373-386.

16. Yang, Y.L.; Deng, X.C.; Xiang, S.W.; Jia, W.S. Well-posedness for a class of strong vector equilibrium problems. J. Nonlinear Sci. Appl. 2016, 9, 1-8.

17. Huang, N.J.; Lan, H.Y.; Cho, Y.J. Sensitivity analysis for nonlinear generalized mixed implicit equilibrium problems with non-monotone set-valued mappings. J. Comput. Appl. Math. 2006, 196, 608-618. [CrossRef]

18. Zhang, L.M.; Hao, Y. Fixed point methods for solving solutions of a generalized equilibrium problem. J. Nonlinear Sci. Appl. 2016, 9, 149-159. [CrossRef]

19. Strodiot, J.J.; Vuong, P.T.; van Nguyen, T.T. A class of shrinking projection extragradient methods for solving non-monotone equilibrium problems in Hilbert spaces. J. Glob. Optim. 2016, 64, 159-178. [CrossRef]

20. Giancarlo, B.; Mauro, P. Descent and Penalization Techniques for Equilibrium Problems with Nonlinear Constraints. J. Optim. Theory Appl. 2015, 164, 804-818. 
21. Huppmann, D.; Siddiqui, S. An exact solution method for binary equilibrium problems with compensation and the power market uplift problem. Eur. J. Oper. Res. 2018, 266, 622-638. [CrossRef]

22. Iusem, A.N.; Mohebbi, V. Extragradient methods for vector equilibrium problems in Banach spaces. Numer. Funct. Anal. Optim. 2019, 40, 993-1022. [CrossRef]

23. Simon, H.A. Administrative Behavior: A Study of Decision-Making Processces in Administrative Organizations, 4th ed.; Mechanical Industry Press: Beijing, China, 2013.

24. Morgan, J.; Scalzo, V. Pseudocontinuous functions and existence of Nash equilibria. J. Math. Econ. 2007, 43, 174-183. [CrossRef]

25. Yu, J. Bounded Rationality and Stability of Equilibrium for Game Theory; Science Press: Beijing, China, 2017.

26. Qiu, X.L.; Jia, W.S.; Peng, D.T. An approximation theorem and generic convergence for equlibrium prlblems. J. Inequalities Appl. 2018, 30, 1-12.

(C) 2020 by the authors. Licensee MDPI, Basel, Switzerland. This article is an open access article distributed under the terms and conditions of the Creative Commons Attribution (CC BY) license (http://creativecommons.org/licenses/by/4.0/). 